\title{
Varroa-Bekämpfung mit Komponenten von ätherischen Ölen
}

\author{
M Rickli, A Imdorf, V Kilchenmann \\ Forschungsanstalt für Milchwirtschaft, Sektion Bienen, $\mathrm{CH}-3097$ Liebefeld, Schweiz
}

(Eingegangen 8 März 1991; angenommen 6 Juni 1991)

\begin{abstract}
Zusammenfassung - Ätherische Öle zeigen oft eine attraktive, repellente oder toxische Wirkung auf Varroa jacobsoni. Ein Produkt auf der Basis von Thymol, Eucalyptol, Menthol und Campher wurde in Feldversuchen getestet. Bei einer Behandlungsdauer von 38 Tagen wurde ein durchschnittlicher Wirkungsgrad von $96,4 \%$, bei einer Dauer von 79 Tagen $99,0 \%$ erzielt. Ob das Produkt sämtlichen Anforderungen genügt, welche wir an eine empfehlenswerte Bekämplungsmethode stellen, wird zur Zeit geprütt.
\end{abstract}

Varroa jacobsoni / Apis mellifera / chemische Bekämpłung / ätherisches Öl / Wirkungsgrad

\section{EINLEITUNG}

Seit dem Auftreten des Bienenparasiten Varroa jacobsoni (Oud) sind Forscher und Imker auf der Suche nach der optimalen Bekämpfungsstrategie. Die bis jetzt entwickelten und erfolgreichen Methoden basieren auf synthetischen Akariziden oder auf Ameisensäure meist kombiniert mit bio-technischen Massnahmen (sogenannte "integrierte Konzepte"). Beide Bekämpfungsweisen haben Nachteile: synthetische Akarizide bergen die Gefahr von Rückständen, integrierte Bekämpfung verlangt einen erhöhten Arbeitsaufwand.

Verschiedene Versuche mit Substanzen ohne die erwähnten Nachteile endeten bisher erfolglos. Die meisten von ihnen weisen einen ungenügenden Wirkungsgrad auf. Einzig ätherische Öle zeigen einen teilweisen Erfolg. Einige pflanzliche Distillate haben repellente Eigenschaften, andere eine gewisse Attraktivität (zB: Bunsen, pers Mitt; Ciavarella, 1989; Hoppe und Schley, 1984; Kraus, 1988). Zwei Präparate wurden bisher auf den Markt gebracht: "Biologic $V^{\circledR "}$ enthält nach Angabe des Herstellers Öle von Labiatae, Anonaceae und Chenopodiaceae. "Apilife/NAR"*) enthält gemäss Hersteller die Inhaltsstoffe Thymol, Eucalyptol, Menthol und Campher. In der vorliegenden Arbeit werden die Ergebnisse von Feldversuchen mit "Apilife/VAR" vorgestellt.

\footnotetext{
* Hersteller: Chemicals LAIF, Via dell'Artigianato 13, Nuova ZAI, 35010 Vigonza (PD).
} 


\section{METHODE}

\section{"Apilife/VAR"-Tafeln}

Es handelt sich um Vermiculit-Tafeln, die beschickt werden mit 18-20 g einer Mischung aus Thymol $(74,1 \%)$, Eucalyptol $(16,0 \%)$, Menthol $(3,7 \%)$ und Campher (3,7\%) (Restmenge: $2,5 \%$ Vermiculit). In GC/MS, GC-NPD und GC-ECDAnalysen haben wir festgestellt, dass keine synthetischen Akarizide in den Tafeln vorkamen.

\section{Bienenvölker}

Die Tests wurden auf 2 Ständen mit je 20 Völkern in Schweizer-Kästen durchgeführt.

\section{Anwendung}

Gemäss Hersteller wird eine Tafel während 2 Wochen im Bienenvolk belassen. Anschliessend wird eine zweite Tafel erneut für 2 Wochen ins Volk gegeben. In unseren Tests wurden die ersten Tafeln nach 2 Wochen durch neue ersetzt. Die zweiten Tafeln blieben auf dem Stand 1 während 24 Tagen in den Völkern, auf Stand 2 während 65 Tagen. Auf Stand 1 wurde die "ApilifeNAR"-Behandlung am 21 September, auf Stand 2 am 21 Oktober 1990 abgebrochen. Die Tafeln wurden gemäss Anweisung des Herstellers auf die Wabenschenkel zwischen Brutwaben und Deckbrett gelegt.

\section{Kontrolle des Milbenfalles}

Eine Woche vor Behandlungsbeginn wurden Kontrollgitter mit Unterlagen in die Bienenkästen eingesetzt. Die aufgefangenen Milben wurden 1 bis 2 mal pro Woche gezählt.

\section{Kontrolle der Wirksamkeit}

Ende Dezember '90 bzw. Anfang Januar '91 wurden 2 Perizin-Behandlungen zur $\mathrm{Be}$ - stimmung der Restpopulation an Varroa durchgeführt. Zu diesem Zeitpunkt waren die Völker brutfrei. Die Völker besetzten im Durchschnitt 8-9 Waben. Der Wirkungsgrad von Perizin beträgt ca $95 \%$ (Ritter et al 1986).

\section{Temperaturen}

Temperaturen während der "ApilifeNAR"Behandlung auf Stand 1: Mittel im August 17,0 $\left(\max 35^{\circ}, \min 3^{\circ}\right)$, Mittel im September $12.9^{\circ}$, $\left(\max 31^{\circ}, \min -2^{\circ}\right)$. Auf Stand 2: Mittel im August $17,4^{\circ}\left(\max 38^{\circ}, \min 3^{\circ}\right)$, Mittel im September $13,1^{\circ}\left(\max 31^{\circ}, \min -2^{\circ}\right)$, Mittel im Oktober $8,2^{\circ}\left(\max 27^{\circ}, \min -6^{\circ}\right)$.

\section{ERGEBNISSE}

Der Verlauf des Milbenfalles vor, während und nach den Behandlungen ist in Abbildung 1 dargestellt. Die Anzahl Milben auf den Unterlagen während der Behandlung mit "Apilife/VAR" sowie während der Perizin-Kontrollen sind in Tabelle | zusammengefasst. Auf Stand 1 ist der Wirkungsgrad von "Apilife/VAR" mit durchschnittlich $96.4 \%$ etwas geringer ausgefallen als auf Stand 2 (99.0\%). Auf Grund der geringen Anzahl an Milben, die während der Kontroll-Behandlungen mit Perizin anfielen, können wir annehmen, dass nur vereinzelt Milben die Behandlungen überlebten.

\section{DISKUSSION}

Auf Stand 2 wurde ein erstaunlich hoher Wirkungsgrad von durchschnittlich $99,0 \%$ mit einer sehr geringen Streuung erzielt (Tabelle I). Diese Wirksamkeit von "Apilife/ VAR" lässt sich mit jener der synthetischen Pyrethroide Fluvalinat und Flumethrin vergleichen (Koeniger und Fuchs, 1988). Die etwas schlechtere Wirksamkeit auf Stand 
Tabelle I. Anzahl Milben während der Behandlung mit "Apilife/VAR" sowie bei Perizin-Kontrolle (Durchschnitt, Minimum, Maximum).

\begin{tabular}{lrrrrrrr}
\hline & \multicolumn{3}{c}{ Stand 1 $(\mathrm{n}=20)$} & \multicolumn{3}{c}{ Stand $2(\mathrm{n}=20)$} \\
& Durchschn & Min & Max & Durchschn & Min & Max \\
\hline Apilife/NAR & 986 & 365 & 1704 & 2453 & 696 & 4509 \\
$\begin{array}{l}\text { Perizin-Kontr 2x } \\
\text { Wirkungsgrad/Efficiency }\end{array}$ & 37 & 3 & 168 & 24 & 6 & 59 \\
"Apilife/NAR" in \% & 96.4 & 91.0 & 99.4 & 99.0 & 97.9 & 99.8 \\
\hline
\end{tabular}

a)

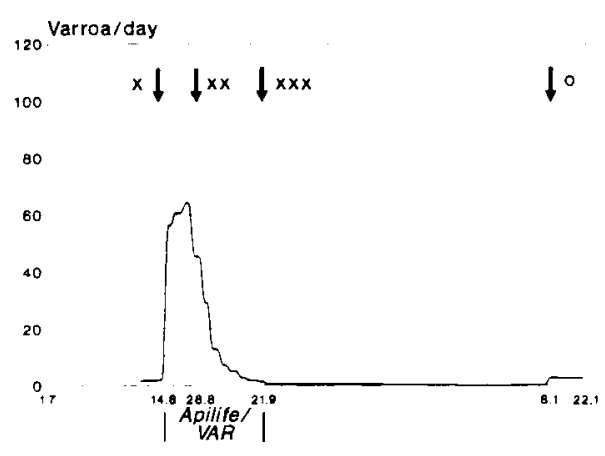

b)

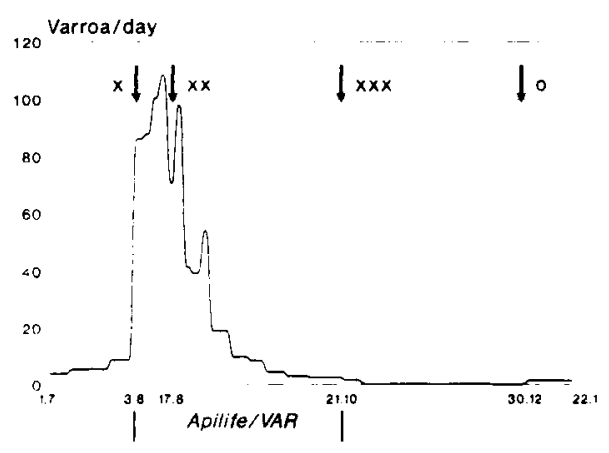

Abb 1. Verlauf des Milbenfalles (Mittelwert aus je 20 Völkern): a) Stand 1: Erste Tafel 14 Tage, zweite Tafel 24 Tage im Volk. b) Stand 2: Erste Tafel 14 Tage, zweite Tafel 65 Tage im Volk. Symbole: $x=$ Beginn der Behandlung; $x x=$ neue Tafeln; $x x x=$ Ende der Behandlung; $0=$ erste von zwei Kontroll-Behandlungen mit Perizin. YAchse = Anzahl Varroa pro Tag.
1 könnte auf die um 41 Tage kürzere Behandlungsdauer zurückgehen. Während dieser Zeit bestand auf Stand 1 die Möglichkeit einer mässigen Rückinvasion der Milben, welche auf Stand 2 wegen der längeren Behandlungsdauer nicht zum Tragen kam.

Es ist uns klar, dass diese guten Behandlungserfolge durch weitere Versuche bestätigt werden müssen, bevor diese Wirkung von "Apilife/VAR" als gesichert gelten kann. An der Rückstands-Problematik wird zur Zeit gearbeitet, Ergebnisse werden später publiziert. Die Wirkungsweise (Toxizität oder Repellent-Effekt) ist unbekannt. Diese Untersuchungen sind in Abklärung. Für andere Milben und für Insekten wurden sowohl Repellent-Wirkung als auch toxische Eigenschaft einzelner Komponenten festgestellt (zB Schearer, 1984; Abivardi und Benz, 1984; Klocke et al, 1987; Larson und Berry, 1984).

Während den Behandlungen haben die Bienen das Futter in den Zellen im Umkreis von ca $5 \mathrm{~cm}$ um die Tabletten herum entfernt. Brutschäden wurden nicht beobachtet und die Völker winterten vor allem auf Stand 2 für schweizerische Verhältnisse sehr stark ein, dh mit 10,000-15,000 Bienen. Räuberei wurde auf keinem Stand beobachtet. Negative Effekte auf das Bienenvolk müssen durch präzise Populationsmessungen überprüft werden. Wir haben vorgesehen, die Plazierung der Ta- 
bletten in den verschiedenen Beutetypen zu optimieren.

\section{DANK}

Dank an Frau A. Burren, Herrn J. Wespi und Dr. A. Kuchen für ihre wertvolle Mitarbeit in diesen Versuchen.

\section{Summary - Treatment against varroa- tosis using compounds of essential} oils. Essential oils have been reported to have some effects on the behavior of Varroa jacobsoni: attracting, repelling or intoxicating the mites. Only few compounds are considered to have an efficiency high enough to be used in bee hives against varroatosis. Our communication reports on the efficiency of a product consisting of thymol $(74.1 \%, w / w)$, eucalyptol (16.0\%), menthol $(3.7 \%)$, camphor $(3.7 \%)$ and vermiculit (2.5\%, carrier substance). We established by means of GC/MS, GCNPD and GC-ECD-analysis, that no other acaricides were present. The pellets, obtained on the market under the name "Apilife/VAR"+) were placed above the brood on the combs in Swiss hives (sideopening). They were exchanged for new pellets after two weeks. The second set of pellets was left in 20 bee colonies for 24 days, in 20 colonies of a second apiary for 65 days. In the first case the treatment ended at the 21st of September, in the second case at the 21st of October 1990. The mites on the hive floor were counted once or twice a week. In December/ January all colonies had 2 controltreatments using Perizin to measure the remaining population of Varroa jacobsoni.
Figure 1 shows the respective numbers of mites per day and colony on the hive floor before, during and after the two treatments. Table $I$ is a summary of the total amount of mites during the treatments with "ApilifeNAR" as well during the two control treatments with Perizin. The tests showed a mean efficiency of the oils of $96.4 \%$ for the shorter treatment and $99.0 \%$ for the longer duration.

Efficiencies like the above found are comparable to those found in treatments using synthetic pyrethroids (Koeniger and Fuchs, 1988). The following questions remain to be solved, before we recommend "Apilife/Var" for general use in varroatosis control: 1 . Is the efficiency consistent? Repetitions of the tests in 1991 will show it. 2. What about residues? 3. What mode of action - repellent or toxic - is involved?

Varroa jacobsoni / Apis mellifera / chemical control / essential oil / efficiency

\section{Résumé - Utilisation de composés d'huiles essentielles contre Varroa. Les huiles essentielles sont connues pour avoir certains effets sur le comportement de Varroa jacobsoni : attraction, répulsion ou intoxication des acariens. On estime que seuls quelques composés ont une efficaci- té suffisante pour pouvoir être utilisés dans les ruches contre la varroatose. Notre tra- vail a porté sur l'efficacité d'un produit con- stitué de thymol $(74,1 \% \mathrm{w} / \mathrm{w})$, d'eucalyptol $(16,0 \%)$, de menthol $(3,7 \%)$, de camphre $(3,7 \%)$ et de vermiculite $(2,5 \%$, support). Nous avons déterminé par GC/MS, GC- NPD et GC-ECD qu'aucun autre acaricide}

+) Manufactured by: Chemicals LAIF, Via dell'Artigianato 13, Nuova ZAI, 35010 Vigonza (PD), Italy. 
n'était présent. Les tablettes, disponibles sur le marché sous la marque "Apilife/ VAR"X) ont été placées au-dessus du couvain dans des ruches suisses (ouverture sur le côté) à raison d'une par colonie. Au bout de 2 semaines, elles ont été remplacées par de nouvelles tablettes, qui ont été laissées 24 jours dans un premier lot de 20 colonies et 65 jours dans un second lot de 20 colonies. Dans le premier cas, le traitement s'est terminé le 21 septembre, dans le second cas le 21 octobre. Les acariens tombés sur le plancher de la ruche ont été dénombrés une ou 2 fois par semaine. En décembre/janvier toutes les colonies ont reçu deux traitements au Perizin pour contrôler la population restante de varroas.

La figure 1 donne les nombres respectifs d'acariens par jour et par colonie présents sur le plancher de la ruche avant, pendant et après les 2 traitements. Le tableau I regroupe le nombre total d'acariens pendant les traitements avec "Apilife/VAR" et les 2 traitements de contrôle avec Perizin. D'après les tests, l'efficacité moyenne des huiles est de $96,4 \%$ pour le traitement court et de $99,0 \%$ pour le plus long.

Ces efficacités sont comparables à celles trouvées avec les traitements qui utilisent des pyréthrinoïdes de synthèse (Koeniger et Fuchs, 1988). II reste à résoudre les questions suivantes avant une utilisation généralisée de ce produit contre la varroatose: 1) l'efficacité est-elle régulière? les répétitions des test en 1991 le montreront. 2) Quid des résidus? 3) Quel est le mode d'action, répulsif ou toxique?

\section{Varroa jacobsoni / Apis mellifera / lutte chimique / huille essentielle / efficacité}

\section{LITERATUR}

Abivardi C, Benz G (1984) New observations on camphor - an old insect repellent - as a relatively safe candidate fumigant against nine insect species. Mitt Schweiz Entomol Ges 57, 179-187

Ciavarella $F$ (1989) Utilisation d'huiles essentielles aromatiques dans la lutte contre Varroa jacobsoni, parasite de l'abeille domestique. Diplôme d'études approfondies, Univérsité d'Aix-Marseille, Oktober 1989

Hoppe H, Schley P (1984) Varroa-Falle mit Lockstoffen möglich? Die Biene 120(9), 387388

Klocke JA, Darlington MV, Balandrin MF (1987) 1,8-cineol (Eucalyptol), a mosquito feeding and ovipositional repellent from volatile oil of Hemizonia fitchii (Asteraceae). J Chem Ecol 13(12), 2131-2141

Kraus B (1988) Effects of chemical compounds in the orientation of Varroa jacobsoni. In: Present status of varroatosis in Europe and progress in the varroa mite control ( $R$ Cavalloro, ed), Proc EC-Experts'Group Meeting, Udine 1988, Italy, 87-92

Koeniger N, Fuchs S (1988) Elf Jahre Erfahrung mit der Varroatose - Rückblick und Aussichten. Allg Dtsch Imkerztg 11/1988, 373-379

Larson KC and Berry RE (1984) Influence of peppermint phenolics and monoterpenes on twospotted spider mite (Acari: Tetranychidae). Environ Entomol 13, 282-285

Ritter W, Perschil F, Jehie B, Koch W und vom Hoevel R (1986) Versuche zur Entwicklung und Prüfung von Perizin, einem systemischen Medikament zur Bekämpfung der Varroatose der Honigbienen. Allg Dtsch Imkerztg 1986/3, 78-82

Schearer WR (1984) Components of oil of tansy (Tanacetum vulgare) that repel colorado potato beetles (Leptinotarsa decemlineata). J Nat Products 47(6), 964-969

x) Fabriqué par Chemicals LAIF, Via dell'Artigionato 13, Nuova ZAI, 35010 Vigonza (PD), Italie. 